\title{
1 Zentrale Begriffe der Pharmakokinetik
}

\author{
Alexander Kratzer
}

\subsection{Definition Pharmakokinetik}

Die Pharmakokinetik stellt ein Teilgebiet der Pharmakologie dar und befasst sich speziell mit Absorption, Verteilung, Biotransformation und Exkretion von Pharmaka im Organismus.

Gemeinsam mit der verabreichten Dosis bestimmen diese Vorgänge die Konzentration eines Wirkstoffs an seinem Wirkort und damit auch das Ausmaß des therapeutischen Effekts sowie auch eventuell auftretender unerwünschter Arzneimittelwirkungen.

Die Pharmakokinetik beschreibt die Wirkung des Organismus auf das verabreichte Arzneimittel. Sie wird folglich stark von physiologischen Unterschieden einzelner Patientengruppen bestimmt. Im Gegensatz dazu beschreibt die Pharmakodynamik den Effekt eines Pharmakons auf den Körper durch Aufklärung der molekularen Wirkmechanismen.

\subsection{Zentrale Größen der Pharmakokinetik}

\subsubsection{Verteilungsvolumen}

Das Verteilungsvolumen $\left(\mathrm{V}_{\mathrm{d}}\right.$, volume of distribution) ist ein Proportionalitätsfaktor, der sich aus der im Blutplasma gemessenen Konzentration ( $\left.c_{\text {plasma }}\right)$ und der verabreichten Menge (D, Dosis) eines Pharmakons nach folgender Gleichung berechnen lässt (s. Abb. 1):
$V_{d}=\frac{D}{C_{\text {Plasma }}}$

Abb. 1 Berechnung des Verteilungsvolumens (Angabe in I oder auch $\mathrm{l} / \mathrm{kg}$ bezogen auf das Körpergewicht)

$V_{d}$ ist also jenes Volumen, welches nötig wäre, um die applizierte Menge eines Pharmakons auf die im Blutplasma gemessene Konzentration zu verdünnen.

Das Verteilungsvolumen ist als fiktiver pharmakokinetischer Parameter anzusehen, der von den physikochemischen Eigenschaften eines Arzneistoffs wesentlich beeinflusst wird.

So sind große, oft vielfach über das tatsächlich vorhandene Plasmavolumen hinausreichende Verteilungsvolumina ein Indiz dafür, dass sich ein Arzneistoff aufgrund seiner physikalischen und/oder chemischen Eigenschaften außerhalb des Blutplasmas in einem sogenannten tiefen Kompartiment verteilt oder sogar anreichert (s. Abb. 2).

Besonders Arzneistoffe, die sehr lipophil sind, weisen häufig hohe Verteilungsvolumina auf, da sie durch ihre Fettlöslichkeit Membranbarrieren durchdringen können und sich auch im Fettgewebe anreichern.

Polare oder ionische Pharmaka sowie Wirkstoffe mit hoher Tendenz zur Bindung an Plasmaeiweiße sind dagegen durch ein niedriges Verteilungsvolumen charakterisiert, da sie durch ihre physiko- 


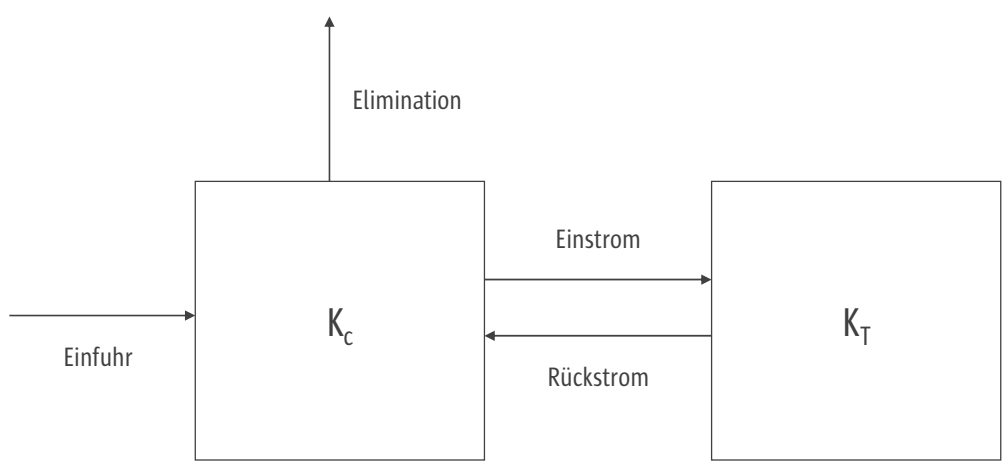

Abb. 2 Schematische Darstellung eines pharmakokinetischen Zweikompartiment-Modells. Viele Arzneistoffe verbleiben nach Applikation nicht im zentralen Kompartiment $K_{c}$ (Blutplasma), sondern verteilen sich im Organismus durch Einstrom in ein sogenanntes tiefes Kompartiment $K_{\mathrm{T}}$ (z.B. Fettgewebe bei lipophilen Arzneistoffen). Daraus resultiert eine erniedrigte Plasmakonzentration im zentralen Kompartiment $K_{c}$ sowie folglich ein erhöhtes Verteilungsvolumen. Bei mehrfacher Applikation bildet sich ein Gleichgewicht zwischen Einstrom in und Rückstrom aus $\mathrm{K}_{\mathrm{T}}$ (steady state). Bei Absetzen einer Medikation kann der oft langsame Rückstrom einen Depoteffekt erzeugen.

chemischen Eigenschaften den Plasmaraum nicht in nennenswertem Ausmaß verlassen können.

\section{Obige Ausführungen können durch zwei Beispiele anschaulich verdeutlicht werden}

Heparin als komplexes lineares Polysaccharid kann durch sein hohes Molekulargewicht und seine negative Ladung kaum durch biologische Membranen diffundieren und bleibt daher nach i.v. Gabe im Wesentlichen im Plasmaraum, was in einem Verteilungsvolumen von ca. 2,8 I (70 kg Patient) resultiert. Dies entspricht annähernd dem tatsächlichen Plasmavolumen.

Diazepam hingegen reichert sich nach Applikation stark im Fettgewebe an, was beim gleichen Patienten ein Verteilungsvolumen von $\mathrm{ca} .77 \mathrm{I}$ bedingt.

Da das Verteilungsvolumen, wie schon erläutert, eine Verbindung zwischen Plasmakonzentration und verabreichter Dosis herstellt, ist diese Größe der zentrale Parameter zur Abschätzung der Initialdosis eines Pharmakons.

\subsubsection{Clearance}

Die Clearance (Cl) stellt ein Maß für die Ausscheidungsgeschwindigkeit eines Pharmakons dar. Konkret entspricht die (Plasma-)Clearance dem Volumen an Blutplasma, das pro Zeiteinheit vollständig vom untersuchten Arzneistoff befreit wird.

Mathematisch errechnet sich $\mathrm{Cl}$ als Quotient aus der Ausscheidungsgeschwindigkeit (ausgeschiedene
Menge des Pharmakons pro Zeiteinheit [dE/dt]) und der Konzentration des Arzneistoffs im Blutplasma $\left(C_{p}\right)$ (s. Abb. 3).

Da an der Clearance eines Arzneistoffes meist mehrere Organe beteiligt sind, ist es möglich, für jedes Ausscheidungsorgan eine organspezifische Clearance zu berechnen.

Maximalwert der Clearance eines Eliminationsorgans ist folglich die Durchblutungsrate, was bedeutet, dass das Blut beim Durchströmen des Eliminationsorgans vollständig vom Arzneistoff befreit wird.

Die Gesamtkörper-Clearance ergibt sich aus der Summe der Clearances der an der Elimination beteiligten Organe. In den meisten Fällen sind nur Leber und Nieren in relevantem Maße an der Ausscheidung eines Pharmakons beteiligt, sodass sich die Gesamtkörper-Clearance in der Regel als Summe der renalen Clearance und der hepatischen Clearance darstellt.

Da die Clearance, wie aus Abbildung 3 hervorgeht, in Abhängigkeit der vorliegenden Plasmakonzentration bestimmt, wie viel eines Arzneistoffs pro Zeiteinheit ausgeschieden wird, stellt sie den zentralen Parameter für die Bestimmung der Erhaltungsdosis bei kontinuierlicher Arzneimittelgabe dar.

$$
\mathrm{Cl}=\frac{\mathrm{dE} / \mathrm{dt}}{\mathrm{C}_{\mathrm{p}}}
$$

Abb. 3 Berechnung der Clearance (Cl) (Angabe in l/min) 
So lässt sich die zur Erhaltung eines bestimmten Plasmaspiegels im steady state $\left(\mathrm{C}_{\mathrm{ss}}\right)$ nötige Dosis pro Zeiteinheit durch Umformung der Gleichung in $\mathrm{Ab}$ bildung 3 folgendermaßen errechnen (s. Abb. 4):

Um eine konstante Plasmakonzentration im steady state aufrecht zu erhalten muss die pro Zeiteinheit zugeführte Erhaltungsdosis an Arzneistoff gleich der in derselben Zeiteinheit ausgeschiedenen Menge [dE/dt] sein.

\subsubsection{Halbwertszeit}

Die Eliminationshalbwertszeit (= Plasmahalbwertszeit) bezeichnet den Zeitraum, in dem die Plasmakonzentration auf die Hälfte ihres ursprünglichen Wertes abfällt.

Die Halbwertszeit stellt aufgrund ihrer Anschaulichkeit und leichten Quantifizierbarkeit heute den in der Medizin am meisten verwendeten pharmakokinetischen Parameter dar. Allerdings darf dabei nicht übersehen werden, dass es sich bei der Halbwertszeit um einen sog. sekundären pharmakokinetischen Parameter handelt. Dieser kann bei Vorliegen einer Eliminationskinetik erster Ordnung (die pro Zeiteinheit ausgeschiedene Menge eines Phar-
$\frac{\mathrm{dE}}{\mathrm{dt}}=\mathrm{C}_{\mathrm{ss}} \times \mathrm{Cl}$

Abb. 4 Errechnung der Dosis pro Zeiteinheit

$\mathrm{t}_{1 / 2}=\frac{0,693 \times \mathrm{V}_{\mathrm{d}}}{\mathrm{Cl}}$

Abb. 5 Abschätzung der Halbwertszeit

makons ist direkt proportional zur Plasmakonzentration), die in den meisten Fällen vorliegt, aus Clearance und Verteilungsvolumen nach der Gleichung in Abbildung 5 abgeschätzt werden.

Die Halbwertszeit kann sich folglich nicht unabhängig verändern. Jede Veränderung von $t_{1 / 2}$ ist Folge einer Veränderung von Clearance und/oder Verteilungsvolumen.

Das Verteilungsvolumen ist der zentrale Parameter zur Abschätzung der Initialdosis. Die Clearance ist der zentrale Parameter zur Abschätzung der Erhaltungsdosis.

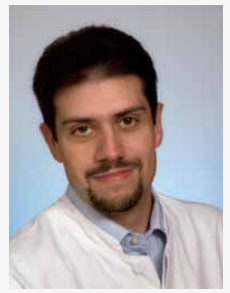

\section{Alexander Kratzer}

Studium der Pharmazie in Regensburg und Erhalt der Approbation 2008. Anschließend Tätigkeit als Klinischer Pharmazeut in der Apotheke der Krankenhäuser des Landkreises Landshut. Seit 2010 am Universitätsklinikum Regensburg tätig als Stationsapotheker auf internistischen und operativen Intensivstationen. Daneben klinisch-pharmazeutische Forschung auf dem Gebiet des Therapeutischen Drug-Monitoring in Kooperation mit dem Institut für Pharmazie Regensburg im Rahmen eines Promotionsprojekts. 